\title{
The Use of CeSo to Improve Turn-Taking Skills among Students with Learning Disabilities
}

\author{
Shahlan Surat ${ }^{1}$, Liana Sadali2 ${ }^{2}$ Saemah Rahman', Saadiah Kummin ${ }^{3}$ \\ ${ }^{1}$ Department of Teaching and Learning Innovation, Faculty of Education, Universiti Kebangsaan Malaysia, Bangi, \\ Malaysia \\ ${ }^{2}$ Bukit Cina Primary School, Malacca, Malaysia \\ ${ }^{3}$ PusatCitra, Universiti Kebangsaan Malaysia, Bangi, Malaysia \\ Email:drshahlan@ukm.edu.my
}

Received 11 January 2016; accepted 25 February 2016; published 29 February 2016

Copyright (C) 2016 by authors and Scientific Research Publishing Inc.

This work is licensed under the Creative Commons Attribution International License (CC BY). http://creativecommons.org/licenses/by/4.0/

(c) (i) Open Access

\begin{abstract}
This research was conducted to minimize the disruptive behavior among the students with learning disability in a special education classroom. Four samples consisting of students with Autism, ADHD, multiple disabilities, Autism-ADHD, and Slow Learner were chosen as research subjects. Selection of the samples was made based on the observation and document analysis. The research methodology is based on the action research model by Lewin (1946) and Laidlaw (1992). CeSo is an abbreviation from the phrase of ceritasosial which refers to social stories in English. The social stories used in this research is entitled Giliran Saya (My turn). The implementation of social story was aided by prompts (microphone) and the elimination of prompts in the intervention. Participants of the study consisted of four students of the schools in the Klang Valley, Malaysia, comprising of students with learning disabilities Autism/ADHD, Autism, ADHD and Slow Learner, aged between 10 - 13 years old. Data were collected using field notes, time sampling record and an interview protocol. The data were analyzed quantitatively and qualitatively using percentage, descriptive and narrative forms. Based on the results, there were reductions in non-turn-taking behavior on all the research subjects. Subjects were able to adapt the turn-taking skills and in turn showed improvement in turn-taking skills. There was also a significant improvement in class control. Through the implementation of CeSo intervention, students were able to understand the situation they faced in a positive manner. This approach is a form of teaching innovations that can be used by teachers in inclusive education in controlling discipline during the process of teaching and learning.
\end{abstract}

\section{Keywords}

Disruptive, Turn-Taking, Autism, ADHD, Slow Learner, Social Stories 


\section{Introduction}

Levin and Nolan (2007) defined disruptive behavior as a disruption in the implementation of teaching and learning. According to Smith \& Smith (2006), disruptive behavior and violence have increased the level of stress and burnout among teachers. Rose \& Gallup (2005) stated that disruptive behavior may be the cause of concern in school systems and that teachers required a method of managing behavior and classroom management. A teacher is under stress when making decisions (Arbuckle \& Little, 2004; Ross et al., 2008; Thompson \& Webber, 2008). Egyed and Short (2006) argued that at times teachers felt stressed and despair with children of learning disabilities and in turn would leave the class and avoid having to deal with these children.

Ross et al. (2008) and Tsouloupas et al. (2010) felt that teachers who left their career as teachers and changed to other careers were due to the behavior of such students. With the awareness of this situation, researchers have chosen to take actions related to disruptive behavior management in order to find alternative solutions that can help these teachers. Disruptive behavior which is focused on this study is the behavior that involves students in turn-taking during the teaching and learning session.

The review and the analysis of the problem are based on the naturalistic or nonparticipant observation using field notes, structured interviews, document analysis (personal and medical records of students) as well as participant observation through data recording using the time sampling record for three times. The focused disruptive behavior is turn-taking behavior.

The findings obtained showed different kinds of disturbing behavior such as annoying their friends, turn-taking, story-telling, talking, shouting and bullying. Findings also indicate that there is frequent disruptive behavior demonstrated by the subjects when the three observations are carried out. Based on the problems discussed, CeSo methods and techniques can help teachers in addressing the problem. CeSo is an abbreviation of the phrase, social stories.

The social story developed by researchers in this study entitled "My Turn" was specially designed to modify the behavior of turn-taking learning disabilities among students with Autism, ADHD or Slow Learner. In an effort to reduce the disruptive behavior of turn-taking, microphones were used as prompts when applying the intervention. This effort has helped the students to adapt the CeSo concept when taking turns. Therefore, this study is conducted to reduce behavioral problem of turn-taking among students with learning disabilities and improve class control during teaching and learning activities.

\section{Literature Review}

Mohd Syahrulikram (2007) states that most of the children were found to have difficulties in the ability to wait, share and take turns. Children who have trouble with communication find it difficult in understanding, taking turns and sharing. K. A. Razhiyah (2008) says that autistic children often have trouble taking turns because they do not understand that in a game or the rules, turn-taking is necessary. They should be taught to take turns and should be shown the right way to take turns.

Jamila (2009) on the other hand commented about children who are diagnosed with Attention Deficit Hyperactive Disorders (ADHD) which can be detected through various symptoms such as the inability to focus, impulsive and hyperactive. A child with ADHD who shows symptoms of impulsive act without thinking, switches from one activity to another activity, finds difficulty in handling any one activity, unable to wait his or her turn should be given more attention. The ability to take turns will enable the children to get basic human needs and the right to be actively involved in classroom and community (DeLuzio \& Girolametto, 2011; Mashford Scott \& Church, 2011).

Apart from that, children with learning difficulties not only require immediate attention but also assistance and instructions on how to understand, respond and take turns during a conversation (Brown, Odom, McConnel, \& Rathel, 2008). The founder of conversation analysis, Sacks (1995), states that the basic unit in conversation is turn-taking which means the flow of the speech. Normally, samples do not talk at the same time and instead wait for their turns. A further study based on Sack's (1995) research by Mohd Syahrulikram (2007) states that taking turns is an important skill in everyday communication. When somebody is talking, another person should be silent and wait for his turn until his friend has stopped talking.

Social stories are simple and short stories to teach certain skills, events, concepts or social behavior to autistic children (Gray, 1998, 2000). The story is written in active sentences using the first person pronoun so that children can easily identify the situations portrayed in the story. Social stories give information about what hap- 
pened, why it happened, who was involved, when a particular event or activity took place and what appropriate responses are expected of children in a given social situation.

The specific procedures in writing social stories are shown by Gray (2000) who uses a combination of verses and sentences (descriptive words to present what had happened; sentences that give individual perspectives such a sentence instructing a students to do something; emphasizing the sentences with emphasis on the social stories) to emphasize on reality. Social Scripts were used to explain the situation clearly, visually where it can be repeatedly referred to in the script. In this regard, we can make it clear to autistic individuals on how they ought to behave (Gray, 1993). The other purpose of the using the social script is to facilitate the teaching of social skills, limiting the behavior from recurring, assisting in keeping track of changes and new teaching routine, describing a person's behavior and reducing the challenging behavior.

Children often require special assistance in the process of behavioral change. The word "prompting" means providing 3D shaped materials in the structure of the assignment. Prompts can be applied with a behavioral intervention (Schloss, 1986). Cooper et al. (in Miltenberger, 2008) states that prompts are used to increase the probability of an individual doing the right behavior at the right time. They are used during training to discriminate individuals to do the right behavior after giving the stimulus.

Prompts are stimuli provided before or during the conduct of a particular behavior. Prompts help to assist the emergence of behavior so that teachers can give reinforcement. Swaggart et al. (1995) preliminary study is the use of social stories to increase the speaking etiquette among girls aged 11 years and play skills of two boys aged 7 years. His findings indicate the occurrence of a reduction in unwanted behavior. However, the use of Antecedent-Behavior $(\mathrm{AB})$ design and the various components of interventions make it difficult to make a clear conclusion about the effectiveness of social story intervention.

A study by Kuttler et al. (1998) showed a decrease in inappropriate behavior of students who tend to drop onto the floor, namely the behavior of rampage when social story intervention was performed. Similarly they did the same thing even when the intervention was withdrawn. Brownell (2002) had adopted the social stories in the form of music by comparing the social stories conducted in the form of reading and in the form of music. The outcome of the studies showed there is an improvement in the behavior of the target subjects when the intervention was carried out both in the form of reading and singing.

It was found that singing is more effective than in the form of reading. However, Crozier et al. (2005) in his study showed that the disruptive behavior of autistic children decreased after using social stories. The effects were studied using the help of verbal and nonverbal prompts. With the help of verbal prompts, the decrease in disruptive behavior was greater. Scattone et al. (2012) found that social stories which are developed effectively and introduced in the natural environment targeting these three autistic children have proven to have a decrease in disruptive behavior when the intervention was implemented to all samples.

\section{Methodology}

\subsection{Research Design}

The study is based on an action research using Lewin (1946) and Laidlaw (1992) model.

1) Practice

Data collection to identify aspects of the practice of seeking to use the improved analysis of documents, field notes posts, sampling time and records, interviews.

2) Planning

Action plans to improve practice. Produce CeSo Intervention (Social Stories).

3) Action Plan

Step 1: the introduction of a social story script "My Turn".

Step 2: introduction of the microphone and its function.

Step 3: using the script and microphone in teaching and learning.

4) Observation

Data collection about the effects of the action through field notes record, a record of time sampling and final interview.

5) Reflection

Reflection was conducted by means of analysis and evaluation, and reflect on the effectiveness of the actions taken based on the data collected. In this study, researchers have only chosen 4 out of 7 students as samples in 
order to pay attention and giving guidance more seriously. Table 1 shows the information of the target group in detail.

\subsection{Implementation of CeSo Intervention}

Implementation of CeSo intervention was carried out by introducing social scripts, followed by the introduction of prompts (using microphone) and the use of both social scripts and microphone in the teaching and learning sessions. The steps of implementing the CeSo intervention are as follows.

Step 1 Introduction to CeSo "My Turn"

Each subject was given a card which contains a social script. The reading activities and understanding the content of the social script was conducted to evaluate on the extent to which the students understood the script. Some questions were directed to the subjects in order to evaluate their understanding of the story presented in the social script. Here are some questions asked by researchers after the subjects have read the social script:

1) Do you all like asking questions to the teachers?

2) If you and your friends make a din, do teachers get to hear your questions?

3) What should you do if the other partner is talking?

4) When can you talk?

5) Would you be happy if teachers listen to what you have to say?

In this Q \& A activity, the teacher associates the social situation in the script with the subjects' situation in class. Below is the implementation of the social story implemented on the students.

My Turn

"I like to ask questions to the teachers, But teachers can't hear my question because my friends and I always are always making noise, I want teachers to listen to what I have to say, We all need to be quiet, When I get a microphone, I will speak, Teachers will hear my question, I'll be happy."

Step 2 Prompts (microphone)

The use of microphone for simple simulation activities. Only students who held the microphone talked while other students remained silent. However, during the teaching and learning session the students responded was on a basis of turn-taking while waiting for their turn to use the microphone.

Step 3 Conditioning

This activity was regularly used in every steps in teaching and was made a routine especially in activities involving discussion and Q \& A. If the turn-taking behavior was demonstrated by the subject, then the researcher would remind the subject of the social script read by other students in the early session of teaching and learning. The rules and routine in the context of classroom behavior are components which have led to the success of classroom organization and management plan, including what is expected, what is the reinforcement, what will be taught again if there was an unwanted behavior (Colvin et al., 1993).

\section{Finding}

Based on the non-participant observations conducted 3 times during the intervention, Figure 1 shows that during the first intervention the subject of $\mathrm{B}$ and $\mathrm{D}$ were able to follow the instructions on the social scripts quite well. Students A was also able to follow the instructions on the social scripts but only for a limited time. On the contrary, students C frequently spoke but kept interrupting his friends. While the second intervention was carried out, it shows the behavior of turn-taking decreased especially for students B, C and d. Moreover, the behavior of students A was still difficult to handle. The third intervention however, showed the behavior of students A gradually improved as opposed to before the intervention was carried out. On the other hand, researchers

Table 1. Information on the subjects of the study.

\begin{tabular}{cccccc}
\hline No. & Research Subject & Gender & Race & Age & Learning Disabilities \\
\hline 1 & Student A & Male & Malay & 13 & Autism/ADHD \\
2 & Student B & Male & Malay & 12 & Autism \\
3 & Student C & Male & Malay & 10 & ADHD \\
4 & Student D & Female & Malay & 11 & Slow Learner \\
\hline
\end{tabular}




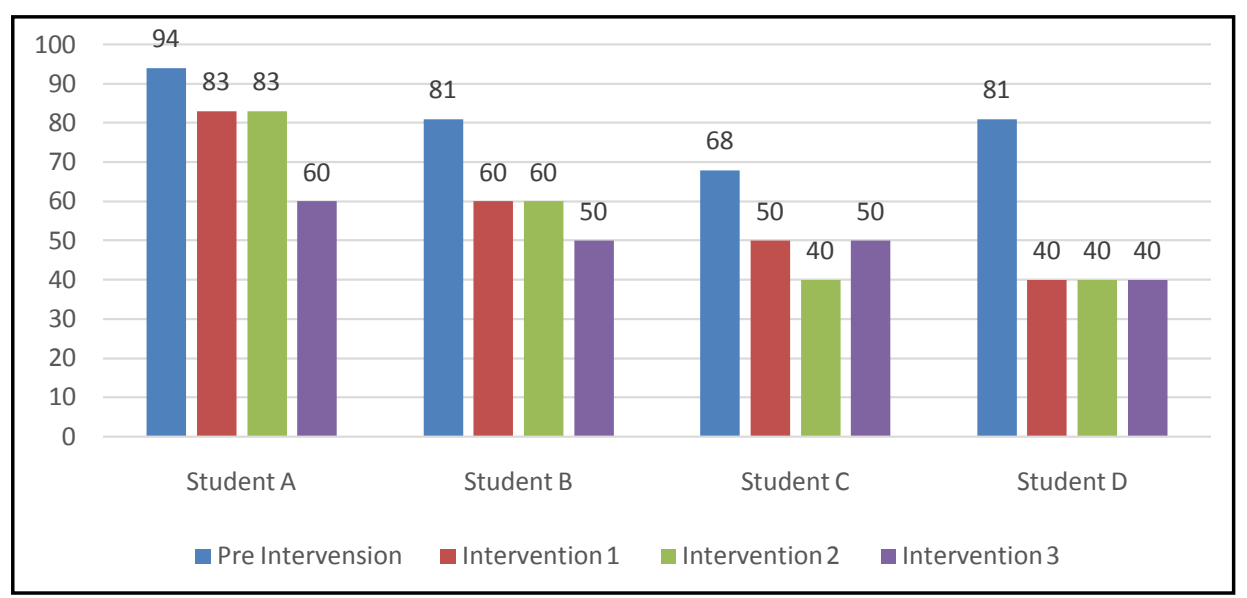

Figure 1. Frequency of turn-taking behavior before and after CeSo intervention.

found students B, C and D are already prone to take turns.

Participant observation was carried out through recording the data using the time sampling record conducted three times during the intervention. The purpose of this observation is to get the change in patterns of the students' behavior every time the intervention was carried out. After the intervention, almost all the subjects showed positive behavior by taking turns when talking. Based on the observation by three teachers, they have agreed that disruptive behavior of turn-taking on Students A, B, C and D showed improvement in taking turns. Apart from observation, structured interview using interview protocols were used to support the data obtained through field notes and time sampling records. All three teachers also agreed that there was an improvement in class control after the implementation of the intervention. Therefore, the findings clearly showed that CeSo intervention succeeded in reducing the disruptive behavior among students with learning disabilities in taking their turns.

\section{Discussion and Implications of the Study}

In conclusion, this study has proven that the use of CeSo will reduce the disruptive behavior of turn-taking of students with learning disabilities. These findings reinforce the studies by Crozier et al. (2005) who used social stories which had been modified to reduce the disruptive behavior of autistic children. Thus, it shows that the social story intervention which have been modified indicates that behavior which can be reduced through CeSo is disruptive, negative and anti-social behavior. The use of the medium of video and audio in the intervention can be implemented to students who have low levels of reading ability.

With verbal cues, the reduction of disruptive behavior was greater. In addition, the findings of this study reinforced the findings of Scattone et al. (2002) who found that social stories which were developed effectively and introduced in the natural environment in autistic children had proven a decrease in disruptive behavior after implementing the intervention on all the subjects of the study. This finding also explains that the problem of disruptive behaviors was beyond the subconscious of the students themselves. The students probably perceived that thoughts and values were considered the same as those people around him. Therefore, the use of CeSo has helped students developed the positive behavior that may help them assimilate in the classroom surrounding.

Besides that, the use of CeSo also helps students to feel empathy and face the situation directly where goals and ways for their positive behavior can be specified clearly. There was a transfer of learning in which students were made aware of the inappropriateness of the turn-taking behavior. CeSo also helped the students to understand that the disruptive behavior had caused discomfort among other students in the class. As a result, positive behavior has resulted in improvement in social skills thus enabling students to take turns in various situations in the classroom. The findings of this study would benefit teachers, individuals and the researcher himself at the outset in addressing students' problem in disruptive behavior. This, at once, became a learning guide to improve teaching practices in the classroom and meet the final goal to develop self-discipline teachers. This is important because the teachers at the beginning of the study were found to have less exposure on aspects of students in disciplinary control. 
Therefore, the gradual elimination of prompts were observed and practiced especially on students with learning disabilities. This is to ensure that the students are not dependent on the use of prompts as special assistance in handling any task. A modification to the CeSo to change the behavior of other disabilities can also be applied. Among the behavior which is suitable in overcoming with the use of CeSo is a disruptive type behavior, negative and antisocial behavior. This shows that the CeSo intervention can change disruptive student behavior through verbal and non-verbal instruction cues among students. Nevertheless, this study is limited to a few students with learning disabilities category of autism, ADHD, and slow leaner. However, the intervention can be extended to student's hyper active to reduce disruptive behavior during the teaching and learning process in the classroom. In addition, it is proposed that the use of CeSo should be expanded in other fields especially on special education students in Malaysia in addition to behavior management as a form of innovation in teaching and learning.

\section{References}

Brown, W. B., Odom, S. L., McConnell, S. R., \& Rathel, J. M. (2008). Peer Interactions for Preschool Children with Developmental Difficulties. In W. B. Brown, S. L. Odom \& S. R. McConnell (Eds.), Social Competence of Young Children with Disabilities: Risk, Disability, and Intervention (pp. 141-164). Baltimore: Paul H. Brookes.

Brown, W., Odom, S. L. \& McConnell, S. R. (2008). Social Competence of Young Children: Risk, Disability, and Intervention. Baltimore: Brookes, 141-163.

Brownell, M. D. (2002). Musically Adapted Social Stories to Modify Behaviors in Students with Autism: Four Case Studies. Journal of Music Therapy, 39, 117-144. http://dx.doi.org/10.1093/jmt/39.2.117

Colvin, G., Kame“enui, E. J., \& Sugai, G. (1993). Reconceptualizing Behavior Management and School-Wide Discipline in General Education. Education and Treatment of Children, 16, 361-381.

Crozier, S., \& Tincani, M. J. (2005). Using a Modified Social Story to Decrease Disruptive Behavior of a Child with Autism. Focus on Autism and Other Developmental Disabilities, 20, 150-157. http://dx.doi.org/10.1177/10883576050200030301

DeLuzio, J., \& Girolametto, L. (2011). Peer Interactions of Preschool Children with and without Hearing Loss. Journal of Speech, Language, and Hearing Research, 54, 1197-1210. http://dx.doi.org/10.1044/1092-4388(2010/10-0099)

Gray, C. (1993). Social Stories: Improving Responses of Children with Autism with Accurate Social Information. Focus on Autistic Behaviour, 8, 1-10.

Gray, C. (1998). Social Stories 101. The Morning News, 10, 2-6.

Gray, C. (2000). The New Social Stories Book. Arlington, TX: Future Horizon.

Jamila, K. A. M. (2009). Pendidikan Khas Untuk Kanak-Kanak Istimewa. Kuala Lumpur: PTS Professional Publishing Sdn. Bhd.

Razhiyah, K. A. (2008). Apa Itu Autisme? Kuala Lumpur: PTS Professional Publishing Sdn. Bhd.

Kuttler, S., Myles, B. S., \& Carlson, J. K. (1998). The Use of Social Stories to Reduce Precursors to Tantrum Behavior in a Student with Autism. Focus on Autism and Other Developmental Disabilities, 13, 176-182. http://dx.doi.org/10.1177/108835769801300306

Levin, J., \& Nolan, J. F. (2007). Principles of Classroom Management: A Professional Decision-Making Model (6th ed.). Boston, MA: Allyn \& Bacon.

Miltenberger, R. G. (2008). Behavior Modification: Principles and Procedures (4th ed.). Belmont, CA: Thompson Wadsworth.

Syahrulikram, M., \& Azizah, N. (2007). Kemahiran Awal Pendengaran Dan Pertuturan. Selangor: Saahifah Global Resources.

Rose, L. C., \& Gallup, A. M. (2005). The 37th Annual Phi Delta Kappa/Gallup Poll of the Public's Attitudes toward the Public Schools. Phi Delta Kappan, 87, 41-57. http://dx.doi.org/10.1177/003172170508700110

Sacks, H. (1995). Lectures on Conversation (2 Vols.). Oxford: Basil Blackwell. http://dx.doi.org/10.1002/9781444328301

Schloss, P. J. (1986). Sequential Prompt Instruction for Mildly Handicapped Learners. Teaching Exceptional Children, 18, 181-184.

Scattone, D., Wilczynski, S. M., Edwards, R. P., \& Rabian, B. (2002). Decreasing Disruptive Behaviors of Children with Autism Using Social Stories. Journal of Autism and Developmental Disorders, 32, 535-543. http://dx.doi.org/10.1023/A:1021250813367

Smith, D. L., \& Smith, B. J. (2006). Perceptions of Violence: The Views of Teachers Who Left Urban Schools. The High School Journal, 89, 34-42. http://dx.doi.org/10.1353/hsj.2006.0004

Swaggart, B. L., Gagnon, E., Bock, S. J., Earles, T. L., Quinn, C., Myles, B. S. et al. (1995). Using Social Stories to Teach Social and Behavioral Skills to Children with Autism. Focus on Autistic Behavior, 10, 1-16. 